\title{
Rezension
}

Elisabeth Heyne*

\section{Depersonalisierte Autobiographien. Zur deutschen Erstübersetzung von Roger Caillois' Der Fluss Alpheios}


Elisabeth Heyne M.A., Institut für Germanistik, Technische Universität Dresden, Strehlener Straße 22/24, D-01069

Dresden, email: elisabeth.heyne@tu-dresden.de

„De quelque façon, chaque écrivain, même le plus secret, ne cesse jamais de rédiger une autobiographie déguisée: son œuvre."1

Der kanarische Drachenbaum (Dracaena draco) ist ein Spargelgewächs. Aufgrund seiner tausendfachen Gabelungen und seines hohen Alters, auf das man nur durch seinen langsamen Wuchs schließen konnte - denn Altersringe hat er nicht - faszinierte er bereits 1799 Alexander von Humboldt während seines Aufenthalts auf Teneriffa. Von Humboldts ausführlicher Beschreibung des Drachenbaums angelockt, träumte auch Darwin von einer Begegnung mit dem Gewächs, die aber blieb inm versagt. Bis ins 20. Jahrhundert und zu den französischen Surrealisten hält sich die Faszination. So reiht sich auch der französische Schriftsteller, Soziologe, Philosoph und Übersetzer Roger Caillois vermittelt über André Bretons Beschreibung des "Baums" in diese Tradition ein. Caillois machte sich über 150 Jahre nach Humboldt auf, einen Drachenbaum zu sehen, und erhob ihn daraufhin zum Emblem seines Denkens und Schreibens. Dies erläutert nun - neben vielen anderen aufschlussreichen Details - die erste deutsche Übersetzung seines autobiographischen Werks Der Fluss Alpheios (Le fleuve Alphée).

Die in Frankreich 1978, im Jahr seines Todes, publizierte Autobiographie Caillois' ist 2016 in einer schön gestalteten Ausgabe bei Brinkmann \& Bose erschienen, präzise übersetzt von Rainer G. Schmidt und herausgegeben von Anne von der Heiden und Sarah Kolb. ${ }^{2}$ Damit wird nach dem Erscheinen von Dissymmetrie 2015 oder Patago-

1 Caillois, Roger (1974): Approches de l'imaginaire. Paris: Gallimard, S. 8.

2 Caillois, Roger (2016): Der Fluss Alpheios. Übersetzt und mit Anmerkungen versehen von Rainer G. Schmidt, herausgegeben und mit einem Nachwort von Anne von der Heiden und Sarah Kolb. Berlin: Brinkmann \& Bose. Alle folgenden Seitenangaben in Klammern beziehen sich auf die genannte Ausgabe. nien und weitere Streifzüge 2016 (auch in einer Übersetzung von Rainer G. Schmidt) die Reihe deutscher Übersetzungen von Texten Caillois' weitergeführt. Diese Übersetzung erfreut allerdings deshalb besonders, weil es sich hier um einen für Caillois außergewöhnlichen Text handelt, der oft als sein zugänglichster beschrieben wurde und in dem er "am Ende seines Wegs, bereits gesundheitlich gezeichnet, auf die Prägungen seiner frühen Kindheit zurückblickt [... $]^{\prime \prime}$. $^{3}$ Bemerkenswert an der deutschen Ausgabe ist die aufwendige und sorgfältige Kommentierung des Textes durch den Übersetzer, die auch solchen Leser*innen, die mit Caillois' Werk nicht vertraut sind, eine Orientierung und Kontextualisierung ermöglicht. Eine hervorragende Einbettung in das Gesamtwerk Caillois' unternimmt zudem das kenntnisreiche Nachwort der beiden Herausgeberinnen. So erleichtert die Ausgabe die oft mühselige Lektüre seiner Texte dadurch, dass ein Blick in die Anmerkungen ausreicht, statt die von Caillois beschriebenen Pflanzen, Steine oder Tiere nachschlagen zu müssen. Durch Einblicke in "Caillois' geheime Elixiere", so der Titel des Nachworts, lässt sich seinen Ausführungen über die „unsichtbaren Analogien" besser folgen. Denn die geheimen, einheitlichen Gesetze des Universums, die Caillois anhand verborgener Analogien zwischen menschlicher Gesellschaft und Kunst, Tier und anorganischer Natur zu greifen versuchte, beschäftigten inn zeitlebens. Mithilfe von Nachwort und Kommentarapparat ist es möglich, in Bezügen, wie etwa auf den emblematischen kanarischen Drachenbaum, „in dessen endloser Verdopplung die Kreuzungen und Verzweigungen der Welt unendlichen Wiederhall finden" (155), ein Modell für sein Denken zu erkennen und Verbindungen mit zentralen Personen des 20. Jahrhunderts wie André Breton nachzuverfolgen.

3 von der Heiden, Anne/Kolb, Sarah (2016): „Caillois' geheime Elixiere". In: Roger Caillois: Der Fluss Alpheios. Berlin: Brinkmann \& Bose, S. 175-186, hier S. 179. 
"[D]ieses seltsame Buch"4, wie es eine zeitgenössische Rezension nennt, trägt zwar zentrale autobiographische Züge, ist aber alles andere als eine klassische Autobiographie: Es berichtet von der Kindheit in Reims, wo Caillois 1913, ein Jahr vor Ausbruch des Ersten Weltkriegs geboren wird. Nach der Evakuierung verbringt er seine ersten Jahre in dörflicher Abgeschiedenheit: "ohne Spielgefährten, ohne Bücher, selbst ohne Bilder, ohne Kino und selbstverständlich ohne Fernsehen" (9). Stattdessen widmet sich der junge Roger den Dingen der Natur, einem „ersten Wissen" (9). Bei der Frage der Entstehung, den Objekten, den einflussreichen Impulsen und der Entwicklung seines Wissens verharrt diese Autobiographie. Es geht Caillois dabei weniger um die Wirkung der Dinge als um die Dinge selbst, um das „Eigenleben der Dinge" (179). Statt der Rekonstruktion eines "klassischen Werdegangs" erkennen die Herausgeberinnen in diesem Text, wie sie im Nachwort vermerken, folglich vielmehr eine "Rückkehr zu den Quellen" (179).

Gemeint ist damit einerseits die Hinwendung zu den dinglichen Ursprüngen seines Wissens. Gegliedert ist Der Fluss Alpheios in zwei Teile, deren Unterkapitel sich solchen Dingen, Bildern, Pflanzen oder Steinen sowie zentralen Denkfiguren seines Werks widmen. Den kritischen und kursorischen Durchgang, den Caillois hier selbst durch sein literarisches und wissenschaftliches Schaffen vornimmt, ergänzt der Kommentarapparat der vorliegenden Ausgabe dankenswerter Weise durch die konkreten Literaturangaben zu den von Caillois jeweils gemeinten Texten. Andererseits verweist die Rückkehr zu den Quellen auf ein mythisches Bild, auf das sich Caillois' autobiographisches Projekt gründet: Alpheios, neugriechisch Alfios, bezeichnet einen Fluss, der in das Ionische Meer mündet und teilweise unterirdisch verläuft. Zugleich ist es der Name des mythischen Flussgottes, der sich in die Nymphe Arethusa verliebt. Um ihm zu entkommen, verwandelt sich die Nymphe in eine Quelle und entflieht in dieser Gestalt quer durch das Mittelmeer nach Sizilien. Alpheios aber verwandelt sich daraufhin in einen Fluss und verfolgt sie, am Grund des Meeres, bis er schließlich, am neuen Ufer angekommen, aus dem Meer entsteigt, um sich mit der Quelle

4 Blot, Jean (1979): Roger Caillois 1913-1978. Commentaire 5/2, S. 121-125, hier S. 121: "ce livre étrange". der Arethusa zu vereinigen. Caillois bedient sich des Mythos, um sein schriftstellerisches und wissenschaftliches Leben als fließende Bewegung zu beschreiben, mittels derer er sich zwar in den "Ozean der Wissenschaften" wirft, seine Wasser aber dennoch wieder vom Meer, das er durchquert, zu trennen vermag. Mit dem Ozean bezeichnet er in diesem Bild "die gewaltige kulturelle Klammer" (175), das Meer des Geschriebenen, der Texte, der Kultur und der Wissenschaft, der gelesenen wie auch der eigenen Bücher, "all dieses gelehrte Geschwätz..." (175), dem er trotz seiner Verlockung am Ende seines Lebens wieder zu entkommen versucht.

Denn was Caillois' "seltsames Buch" schließlich doch wieder mit der Autobiographietradition des 20. Jahrhunderts verbindet, ist, dass es sich als eine Geschichte der eigenen Alphabetisierung lesen lässt. Wie in Sartres Les mots oder Elias Canettis Die gerettete Zunge nimmt das Lesenlernen eine wichtige Stellung ein. Allerdings ist es hier gerade der "ungewöhnliche Aufschub, den ich heute für einen Segen halte" (36), der es dem Caillois'schen Alpheios ermöglicht, dem Meer der Alphabetisierung wieder zu entsteigen und der zum zentralen Thema des Buches avanciert: Ersehnte er als Kind zwar fieberhaft, in die Welt der Lesenden aufgenommen zu werden, lernte er durch die Folgen des Kriegs erst spät lesen und auch dann nur über den Umweg auswendig gelernter Eigennamen, denn das Alphabet wurde ihm zunächst nicht beigebracht: "Ich wurde eine Art gebildeter Analphabet" (33). Durch diese späte Prägung verlor sich der Fluss nicht im Ozean und die "Wurzeln und Fasern, die aus meinem alten Wissen kamen" (37), setzten sich rebellisch fort. Zwischen Text und Ding schreibt Der Fluss Alpheios die Kindheitsgeschichte seiner Wissenschaft, die zeitlebens untersucht, wie die Worte und die Dinge zusammenhängen, und dazu den handgreiflichen Dingen jenseits des Textparadigmas nachspürt. So ist die Referenz auf den Fluss letztlich weniger als Bezug auf den (textuellen) Mythos als auf den unterirdischen Fluss als Ding der Natur zu verstehen.

Seltsam ist das Buch aber vor allem, weil es im Modus der Autobiographie versucht, die eigene Autorfigur zu tilgen. Diese Tendenz erlaubt es, einen Bogen zu spannen zwischen diesem letzten und dem ersten Buch, das der gerade 20-jährige Caillois zwischen 1933 und 1935 verfasste, allerdings nie selbst publizierte. Erst 1981, drei Jahre 
nach seinem Tod, wurde La nécessité d'esprit (Die Notwendigkeit des Geistes) erstmals veröffentlicht. Die im Alpheios längst verschwundene Autorperson lässt sich in diesem frühen Text etwas deutlicher erahnen: Anhand der wissenschaftlichen Untersuchung seiner experimentellen Texte, die aus eigenen "psychasthenischen" Erfahrungen entstanden und Einblicke in teilweise bewusst herbeigeführte psychische Krisenzustände geben, entwickelte er darin bereits die zentralen Themen seines Werkes. Die eigenen deliranten, poetischen Texte und Gedichte aber tilgte er aus allen späteren Veröffentlichungen: Sein sicherlich berühmtester Text, der 1934 in der surrealistischen Zeitung Minotaure erschienene Aufsatz über die Gottesanbeterin, enthält sie schon nicht mehr, obwohl er implizit auf sie verweist. Erst das posthume La nécessité d'esprit, dessen fünftes Kapitel aus dem Gottesanbeterinnen-Aufsatz besteht, setzt diesen in den größeren Zusammenhang der auto-pathographischen Texte und füllt die Leerstelle der Autorperson paradoxerweise mit Texten, die gerade von Erfahrungen der Depersonalisierung berichten - und dies in Ich-Perspektive. Diese von Anfang an prekäre autobiographische Perspektive wird im Fluss Alpheios ganz durch den wissenschaftlichen, (de- oder) entpersonalisierten Diskurs ersetzt. So bleibt die Depersonalisierung ein zentrales Thema Caillois': Als Forschungsobjekt wie auch auf das eigene Schreiben und zudem das eigene Lesen bezogen.

Auf die Lektüre gewendet taucht diese Bewegung im Fluss Alpheios besonders deutlich in der Analphabetisierungsszene auf, als Denkfigur des
Zeichens ohne Referenz. Als eine solche Figur spielt sie außerdem im gesamten Werk Caillois' eine entscheidende Rolle: Wie in den Steinen, die Caillois sammelt und mit denen er sich exzessiv beschäftigt. Sie sind für inn letztlich Archive, in denen sich nichts lesen lässt, die auf nichts verweisen (60). Wie in den unendlichen Verzweigungen und Verweisungen des Drachenbaums, dessen Alter ein Menschenleben weit übersteigt. Oder wie im Bild des umgekehrten Flusses, der schließlich die Nymphe vergisst, der er nachgejagt ist, um in einer Kluft zu verschwinden: "eine umgekehrte Quelle, eine, die verschluckt" (158). Skizziert Caillois hier das Bild einer Welt ohne Mensch, jenseits des Menschen, nach dem Menschen, das Ende des Anthropozäns, so tut er dies dennoch wieder im Medium des Textes: Eine "Lächerlichkeit", derer er sich bewusst ist: „Ich kann damit leben" (158).

\section{Literaturverzeichnis:}

Blot, Jean (1979): Roger Caillois 1913-1978. In: Commentaire 5/2, S. 121-125.

Caillois, Roger (1974): Approches de I'imaginaire. Paris: Gallimard.

Caillois, Roger (2016): Der Fluss Alpheios. Übersetzt und mit Anmerkungen versehen von Rainer G. Schmidt, hg. u. mit einem Nachwort v. Anne von der Heiden und Sarah Kolb. Berlin: Brinkmann \& Bose.

von der Heiden, Anne/Kolb, Sarah (2016): Caillois' geheime Elixiere. In: Roger Caillois: Der Fluss Alpheios. Berlin: Brinkmann \& Bose, S. 175-186. 